\title{
A Mesenteric Enteric Duplication Cyst Communicating with Jejunum in Adolescent: Communicating Jejunal Duplication Cyst
}

\author{
Keyur Bhatt ${ }^{1}$, Dhaval Mangukiya ${ }^{1}$, Krishna Parekh $^{2}$ \\ ${ }^{1}$ Department of GI \& Minimal Access Surgery, SIDS Hospital \& Research Centre, Surat, India, ${ }^{2}$ Department of Clinical Research, \\ SIDS Hospital \& Research Centre, Surat, India.
}

\section{Corresponding Author: \\ Dr Keyur Bhatt \\ Email: drkeyurbhatt@gmail.com}

This is an Open Access article distributed under the terms of the Creative Commons Attribution License (creativecommons.org/ licenses/by/3.0).

Received Accepted Published

July 7, 2019

December 4, 2019

January 10,2020

\begin{abstract}
Background: Enteric duplication cysts are congenital malformation of gastrointestinal tract which may arise anywhere in alimentary tract. We report a case of symptomatic presentation of communicating jejunal duplication cyst in an adolescent. Case Report: A 14 year old boy presented with persistent abdominal pain and vomiting. $\mathrm{CT}$ abdomen showed communicating jejunal duplication cyst. The duplication cyst was resected with involved segments. Conclusion: Duplication cyst should be considered in differential diagnosis of abdominal cystic lesions.
\end{abstract}

Keywords: Abdominal Pain, Cysts, Gastrointestinal Tract, Tomography, Vomiting.

\section{Introduction}

Enteric duplication cysts are congenital malformation of gastrointestinal tract which may raise anywhere in alimentary tract and presents with various symptoms depending upon the size, shape and structure. This anomaly is most commonly observed in ileum $(30 \%)$, followed by the esophagus $(20 \%)$, colon $(13 \%)$, jejunum $(8 \%)$, stomach (8\%), and duodenum (5\%). Typically, duplication cysts are non-communicating and remains completely isolated from bowel [1]. Our extensive literature search revealed that the communicating duplication cysts are rare and jejunal communicating duplication cysts are even few and far between entity. The overall incidences of duplication cyst are two or three cases per year in pediatric which shows to be 1:4500 births. Besides, this condition can be diagnosed at any age, but vast majority of patients are presented during their infancy $(<2$ years) [2]. We report a case of 14-yearold boy with communicating jejunal duplication cyst.

\section{Case Report}

A 14-year-old boy presented with the complaints of persistent abdominal pain and non-bilious vomiting. He had surgical history of Meckel's diverticulectomy two months back. On examination, the patient had tender abdomen without guarding over epigastrium and left hypochondrium. An abdominal contrast enhanced computed tomography showed fluid filled $(10 \mathrm{~cm}$ long) thick walled, tubular structure in left iliac fossa in mesenteric border adjacent to jejunal loops with $6 \mathrm{~mm}$ wall thick tubular structure, suggestive of communicating duplication cyst [Fig.1]. Hence, exploratory laparotomy was planned to treat communicating duplication cyst. On surgical exploration, a large $10 \times 3 \mathrm{~cm}$ fluid filled tubular bowel like mass with its blind end near duodenojejunal flexure extending up to $10 \mathrm{~cm}$ distal was observed. The tubular mass was parallel to the jejunum within the mesentery and communicating with jejunum till the distal end [Fig.2a]. Therefore, in view of jejunal communication and dense 
adherence with mesentery, it was decided to resect the duplication cyst with involved segments. The infant feeding tube was passed through the fistulous opening of the cyst which revealed communication with the jejunum [Fig.2b]. The histological examination of resected tubular mass had features of duplication cyst. Patient recovered without any post-operative complications.

\section{Discussion}

Enteric duplication cyst is hollow, epitheliumlined, cystic, spherical or tubular structure that is usually attached to the wall of gastrointestinal tract (often share the serosa) and supplied by a common mesenteric blood vessel. This anomaly affects only $0.2 \%$ of adolescents with slight male predominance. Most commonly, it is diagnosed incidentally in young children as it remains asymptomatic [1,2]. In contrast to that our adolescent patient was symptomatic. Duplication cyst have typical phenotypic characteristics: cyst contains mucosa of gastrointestinal tract from where it originates, surrounded by smooth muscles and share a common wall of anatomic site where it is found. In most of the cases, duplication cyst is found on the mesenteric border and diverticulum is found on anti-mesenteric border [1,2]. In our case, the patient had both anomalies on mesenteric border duplication cyst and on anti-mesenteric border Meckel's diverticulum.

This anomaly is most commonly involved with small intestine (44\%) of which two third of the cases are found in ileum and one third cases are found in jejunum. This ratio itself shows rarity of jejunal duplication cyst [3]. Structurally, duplication cysts are either cystic or tubular. Usually $80 \%$ of duplication cysts are spherical and do not communicate with the adjacent bowel lumen. Only $20 \%$ of duplication cysts are tubular and directly communicates with bowel lumen. According to the blood supply to the duplication cyst, it is classified into type I (parallel) which is more towards mesentery with separate blood supply
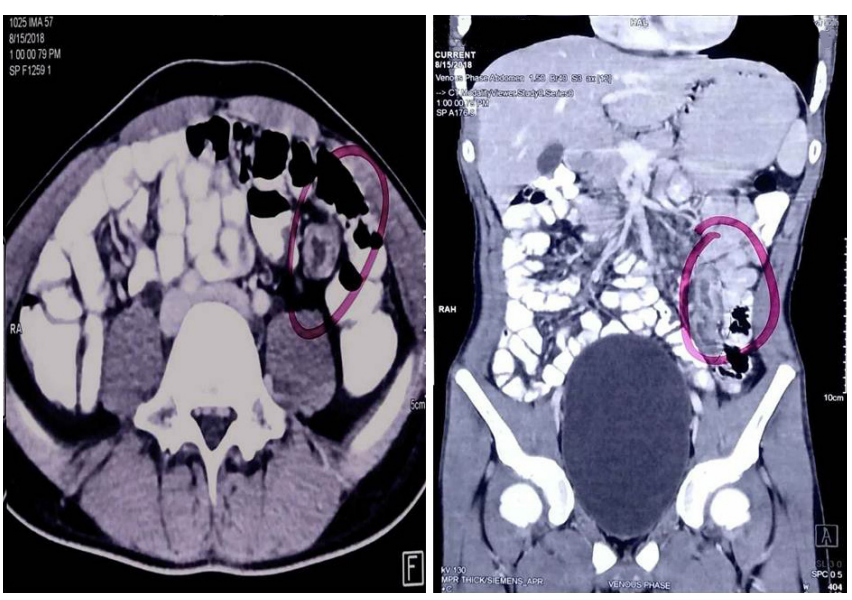

Fig.1: Abdominal contrast computed tomography examination: A large tubular structure on mesenteric border adjacent to jejunum.
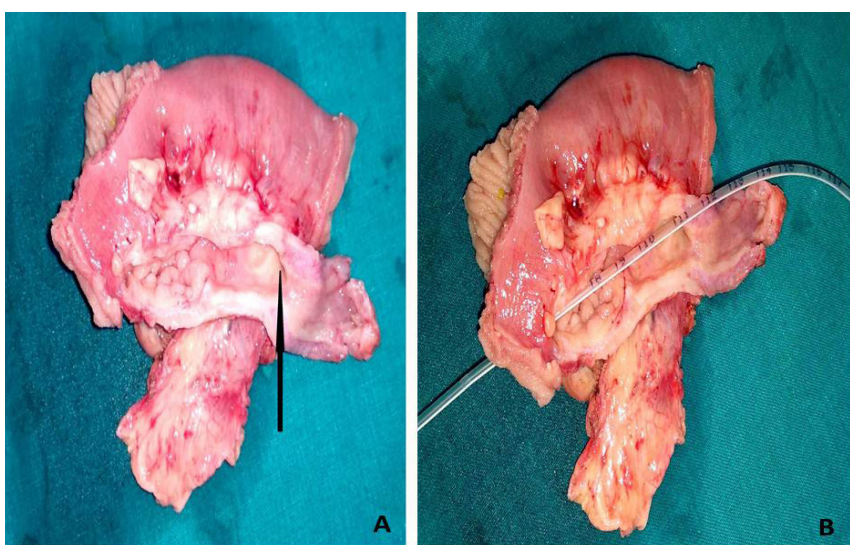

Fig.2: Jejunal duplication cyst resected segment (a): The black arrow showing tubular duplication mesenteric cyst parallel to the jejunum (b): Infant feeding tube is passed which shows communication between cyst and small bowel.

from native bowel and type II (intra-mesenteric) which is cantered in mesentery having vessel both the sides [3]. In our case, duplication cyst rested in mesenteric border with a common pedicle and runs parallel to the luminal communication with the adjacent jejunum.

\section{Conclusion}

This anomaly is usually diagnosed incidentally in young children. Hence, duplication cyst should be considered while examining abdominal cystic lesions. 
Contributors: KB: manuscript editing, and patient management; DM: reviewing the literature, manuscript editing and patient management; KP: critical inputs into the manuscript and manuscript writing. KB will act as a study guarantor. All authors approved the final version of this manuscript and are responsible for all aspects of the study.

Funding: None; Competing interests: None stated.

\section{References}

1. Sharma, S, Yadav AK, Mandal AK, Zaheer S, Yadav DK, Samie A. Enteric duplication cysts in children.
A clinicopathological dilemma. J Clin Diagn Res. 2015;9(8):EC08-11.

2. Nebot CS, Salvador RL, Palacious EC, Aliaga SP, Pradas VI. Enteric duplication cysts in children: varied presentations, varied imaging findings. Insights Imaging. 2018;9(6):1097-1106.

3. Almatrfi, A, Neazy A, Alharbi W, Abokrecha A. Two different presentations of intestinal duplication cyst in pediatric age group. Saudi Surgical Journal. 2018;6(3):100. 